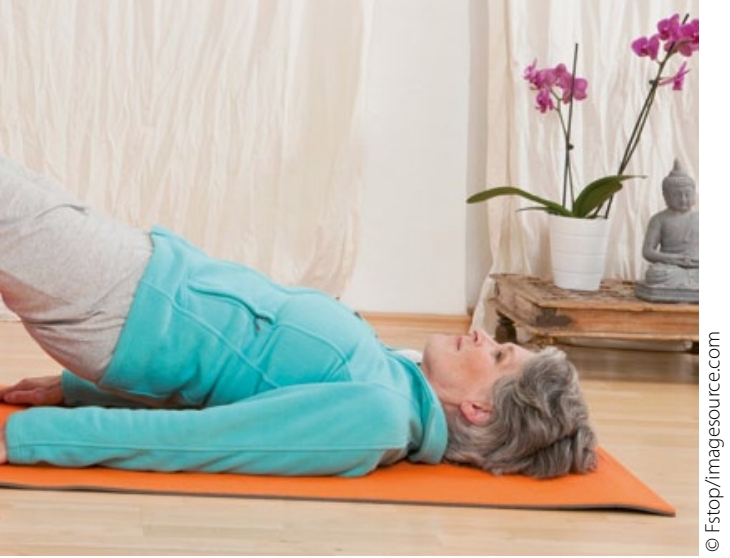

trotz zahlreicher Studien nur schwer treffen, denn die Verfahren wurden meist nicht allein, sondern im Rahmen eines multimodalen Therapieansatzes untersucht. Zudem unterscheiden sich oftmals die Übungsprotokolle und Out-
come-Variablen erheblich, weshalb die Studien kaum vergleichbar sind. Dennoch haben die Entspannungstechniken mittlerweile auch Eingang in verschiedene Leitlinien gefunden, wie etwa in die Nationale Versorgungsleitlinie Kreuzschmerz von 2010 (www.kreuzschmerz.versorgungsleitlinien.de) oder in die S3-Leitlinie der AWMF zur Fibromyalgie (2008).

\section{So lernt es sich leichter}

Wichtig ist, die Erwartungshaltung der Patienten zu relativieren. Viele setzen sich enorm unter Druck und verspannen dadurch noch mehr. Außerdem ist der Patient darüber aufzuklären, dass es sich bei der Entspannung um eine sehr individuelle Körperreaktion handelt und sich der Erfolg nicht darin niederschlägt dass man sich danach besonders entspannt fühlt. Vielen fällt es anfangs nicht leicht, sich zu konzentrieren. Sie sind durch ihre Beschwerden abgelenkt und können nur schwer den Instruktionen folgen. Tägliches Üben erhöht die Effektivität und ermöglicht es den Schmerzpatienten, sich auch in Alltagssituationen zu entspannen und den Schmerz in seine Schranken zu verweisen.

DR. DAGMAR KRAUS

\footnotetext{
- Diezemann A. Entspannungsverfahren bei chronischen Schmerzen. Schmerz 2011 25:445-453

Buschmann K. Psychologische Behandlung bei chronischen Kopf- und Gesichtsschmerzen. 2007: 21:167-179
}

\title{
Entspannungstechniken in der Praxis
}

\section{„Raus aus der Passivität}

\section{Entspannungstechniken haben für Dipl.-Psych. Charles Kimelman, Schmerzpsychotherapeut an der Schmerzklinik des Caritasklini- kums Saarbrücken, seit langem einen festen Platz im multimodalen Schmerztherapiekonzept.}

MMW: Für welche Schmerzpatienten sind Entspannungstechniken geeignet? Kimelmann: Grundsätzlich profitiert jeder Schmerzpatient davon, selbst wenn seine Schmerzen einen somatischen Schwerpunkt haben. Bei den einen werden Entspannungstechniken als flankierende Maßnahme eingesetzt, bei den anderen sind sie Kernstück der Therapie. Letzteres trifft vor allem auf Patienten mit chronischen Rückenschmerzen, Kopfschmerzen oder Fibromyalgie zu.

MMW: Welche Techniken präferieren Sie? Kimelmann: Meine Lieblingsmethoden sind die klinische Hypnose und die Hypnotherapie. Diese Verfahren lassen sich selbst mit Schwerstkranken problemlos durchführen, und sie entfalten eine antinozizeptive Wirkung auf spinaler Ebene. Schmerztherapie wird für den Patienten somit direkt spürbar. Die Schmerzen lassen meist innerhalb einer Sitzung deutlich nach. Das ist mit anderen Verfahren so gezielt nicht möglich. Ein weiterer wichtiger Punkt: Ich kann dem Schmerzpatienten nicht nur bei seinem Schmerz helfen, sondern gleichzeitig psychische Komorbiditäten mitbehandeln, wie Ängste, Depressionen oder Schlafstörungen. Ich arbeite aber auch gerne mit modernen Biofeedback-Methoden oder mit Techniken der Imagination. Inzwischen ist auch die progressive Muskelentspannung ein gängiges Verfahren sowie die verschiedenen meditativen Techniken, die unter dem Begriff der "Achtsamkeit" von sich reden machen.

MMW: Wie lässt sich die Akzeptanz bei den Patienten verbessern?

Kimelmann: Es ist von Vorteil, in einem Team zu arbeiten, in dem alle Kollegen über Entspannungstechniken Bescheid wissen. Im Rahmen der sog. Edukation wird dem Patienten erklärt, was das biopsychosoziale Schmerzkonzept überhaupt ist und wieso es sich bei der Schmerztherapie um einen Lernprozess handelt. Ganz entscheidend ist, den

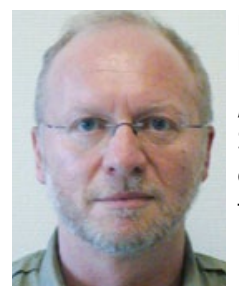

Dipl.-Psych. Charles Kimelman „Entspannung macht Schmerzpatienten gelassener und dickfelliger."

Patienten aus seiner passiven Rolle zu befreien und zu betonen, wie wichtig das eigene Tun für die Schmerzbekämpfung ist.

MMW: Wie wirken Entspannungstechniken? Kimelmann: Eine wichtige Wirkung dieser mentalen Methoden ist die auf das vegetative Nervensystem. Der Patient lernt Kontrolle über Muskel- und Gefäßspannung, also allgemein über die Körperregulation zu bekommen. Zudem erzielt man eine affektive Indifferenz, $d$. h. die Patienten werden gelassener oder - wenn man so will - dickfelliger. Nicht zu verkennen ist auch die mentale Frische, die nach dem Üben einsetzt, das Gefühl des „Ausgeruht-Seins". Das ist vor allem deshalb wichtig, da viele Patienten mit Regenerationsproblemen und Schlafstörungen zu kämpfen haben. Außerdem erhöhen sich die Wahrnehmungsschwellen: Die Patienten werden weniger ablenkbar und störbar. Mit der Hilfe zur Selbsthilfe verbessert sich auch die Selbstwirksamkeit und die Resilienz, die wichtig zur Gesunderhaltung sind. - Interview: Dr. Dagmar Kraus 